\title{
Bacteria-mediated reactivation of gammaherpesviruses
}

\author{
Terry Morris ${ }^{1 *}$, Fang Gu', RoShaunna Rothwell ${ }^{4}$, Jennifer Webster-Cyriaque ${ }^{1,2,3,4}$ \\ From $12^{\text {th }}$ International Conference on Malignancies in AIDS and Other Acquired Immunodeficiencies \\ (ICMAOI) \\ Bethesda, MD, USA. 26-27 April, 2010
}

Significant morbidity is associated with the synergistic and inhibitory interactions of bacteria, viruses, parasites, and fungi. The oral cavity, gut, and genitourinary tract are home to many of these organisms and are the site of virus-associated malignancies that affect millions worldwide. Yet little is known with regard to the cellular and molecular interactions of viral pathogens with the normal flora as well as the interactions among pathogens themselves. Our laboratory is interested in understanding the role of factors present within the immediate environment that may influence reactivation of persistent infection and pathogenesis. Our central hypothesis is that viral-bacterial interactions foster enhanced pathogen replication and modulation of the immune response in the mouth, GI tract, and genito-urinary tract. The detection of replicating virus in these tissues has incited investigation into the relationship between bacterial infection and herpesviral reactivation. We hypothesized that bacterial end-products including short chain fatty acids (SCFA), lipopolysaccharide (LPS), and lipoteichoic acid (LTA) secreted by oral bacteria initiate viral reactivation from latency. Latently infected EBV, KSHV, and MHV 68 cell lines were incubated with crude spent media containing secreted SCFA, and components of bacterial pathogens (E. faecalis, Bacteriodes, Prevotella, Porphomonas, and Fusiobacterium Nucleatum). Cells were then assayed for viral promoter activation, promoter-protein interactions, and state of infection. Following incubation with crude spent media, viral immediate early promoters were activated, the viral early genes were upregulated as determined by RT PCR and western blot, and linear genomes were detected. HDAC inhibition activity as well as protein kinase $\mathrm{C}$ activity increased significantly following treatment with bacterial spent media. KSHV and EBV were consistently reactivated by bacterial metabolites but the mechanism of reactivation was both bacteria, virus, and cell type specific. Interestingly, EBV was preferentially reactivated following toll like receptor stimulation while KSHV and HSV-1 reactivation occurred following HDAC inhibition. In conclusion, these studies provide significant insights to gammaherpesreactivation that may occur in vivo via pathogen-pathogen interaction.

\section{Acknowledgements}

This work was supported by NIDCR OHARA 1 U01 AI068636-01, AAE fellowship to Dr. Gu, and NIH F31 GM070115-02 to Dr. Rothwell. This article has been published as part of Infectious Agents and Cancer Volume 5 Supplement 1, 2010: Proceedings of the $12^{\text {th }}$ International Conference on Malignancies in AIDS and Other Acquired Immunodeficiencies (ICMAOI). The full contents of the supplement are available online at http://www.biomedcentral.com/1750-9378/5?issue=S1.

\section{Author details}

${ }^{1}$ Lineberger Cancer Center, University of North Carolina, Chapel Hill, NC, USA. ${ }^{2}$ Division of Infectious Disease, University of North Carolina, Chapel Hill, NC, USA. ${ }^{3}$ Department of Microbiology and Immunology, University of North Carolina School of Medicine, Chapel Hill, NC, USA. ${ }^{4}$ Department of Dental Ecology, University of North Carolina School of Dentistry Chapel Hill, NC, USA.

Published: 11 October 2010

doi:10.1186/1750-9378-5-S1-A6

Cite this article as: Morris et al:: Bacteria-mediated reactivation of gammaherpesviruses. Infectious Agents and Cancer 2010 5(Suppl 1):A6. 